\title{
LIPOTOXICIDAD EN MÚSCULO ESQUELÉTICO Y SU RELACIÓN CON LA RESISTENCIA INSULÍNICA. ESTUDIOS EN UN MODELO EXPERIMENTAL DE SÍNDROME METABÓLICO
}

\author{
SKELETAL MUSCLE LIPOTOXICITY AND ITS RELATIONSHIP WITH \\ INSULIN RESISTANCE. STUDIES IN AN EXPERIMENTAL MODEL \\ OF METABOLIC SYNDROME
}

María Eugenia Oliva ${ }^{1,2}$, María del Rosario Ferreira ${ }^{1,2}$, Victoria Aiassa ${ }^{1}$, María Eugenia D'Alessandro ${ }^{1,2}$

\section{RESUMEN}

Introducción: el acúmulo de lípidos en el músculo esquelético se encuentra estrechamente vinculado con el desarrollo de la resistencia insulínica. Esta última cumple un rol patogénico central en el desarrollo de numerosos desórdenes metabólicos incluidos en el síndrome metabólico.

Objetivos: analizar algunas vías metabólicas implicadas en el acúmulo de lípidos en el músculo esquelético y su asociación con la resistencia insulínica en un modelo experimental que mimetiza el fenotipo del síndrome metabólico humano.

Materiales y métodos: ratas macho Wistar recibieron una dieta control (DC) o una dieta rica en sacarosa (DRS) durante seis meses. Al final del período experimental se analizó en músculo esquelético gastrocnemio: contenido de triglicéridos (TG), acil-CoA de cadena larga y diacilglicerol, actividad enzimática carnitina palmitoil transferasa muscular (M-CPT1, M-CPT2 y M-CPT total) y masa proteica del PPAR $\alpha$, AMPK y AMPKp. Se determinaron los niveles séricos de TG, AGNE, glucosa, insulina, TNF $\alpha$ y adiponectina. La sensibilidad insulínica se midió por la técnica clamp euglucémica-hiperinsulinémica.

Resultados: en los animales alimentados con DRS la dislipemia, hiperglucemia moderada, insensibilidad insulínica e incremento del contenido de especies lipídicas en el músculo esquelético se acompañaron de una disminución en la actividad enzimática M-CPT1 y M-CPT total, y un descenso de la masa proteica del PPAR $\alpha$. Además se observó una reducción de la masa proteica de la AMPKp, la cual se correlacionó con bajos niveles de adiponectina y elevados niveles de TNF $\alpha$ séricos.

Conclusiones: los resultados aportan nuevos datos sobre algunos mecanismos involucrados en el desarrollo de la lipotoxicidad en el músculo esquelético en ratas dislipémicas insulinorresistentes.

Palabras clave: síndrome metabólico; dieta rica en sacarosa; músculo esquelético; resistencia insulínica; lipotoxicidad.

Revista de la Sociedad Argentina de Diabetes 2019; Vol. 53 (53-62)

\section{ABSTRACT}

Introduction: the skeletal muscle lipid accumulation is closely linked to the development of insulin resistance. The latter plays a central pathogenic role in the development of numerous metabolic disorders included in the metabolic syndrome.

Objectives: to analyze some metabolic pathways involved in the skeletal muscle lipid accumulation and its association with insulin resistance in an experimental model that mimics the phenotype of the human metabolic syndrome.

Materials and methods: male Wistar rats received a control diet (CD) or a sucrose rich diet (SRD) for six months. At the end of the experimental period, in gastrocnemius skeletal muscle were analyzed: triglyceride (TG), long chain acyl-CoA and diacylglycerol (DAG) contents, muscle carnitine palmitoyl transferase enzymes activities (M-CPT1, M-CPT2 and total M-CPT) and protein mass levels of PPAR $\alpha, A M P K$ and AMPKp. Serum levels of TG, AGNE, glucose and insulin, TNF $\alpha$ and adiponectin were determined. Insulin sensitivity was measured by the euglycemic-hyperinsulinemic clamp technique.

Results: in SRD fed animals, dyslipidemia, moderate hyperglycemia, insulin insensitivity and the increased content of lipid species in the skeletal muscle were accompanied by a decrease in the enzymes activities of both M-CPT1 and total M-CPT and protein mass levels of PPAR $\alpha$. In addition, a reduction in the protein mass levels of AMPKp was observed, which was correlated with low serum levels of adiponectin and high levels of TNFo.

Conclusions: the results provide new data on some mechanisms involved in the development of lipotoxicity in skeletal muscle in insulin resistant dyslipidemic rats.

Key words: metabolic syndrome; sucrose rich diet; skeletal muscle; insulin resistant; lipotoxicity.

Revista de la Sociedad Argentina de Diabetes 2019; Vol. 53 (53-62)
1 Laboratorio de Estudio de Enfermedades Metabólicas Relacionadas con la Nutrición, Facultad de Bioquímica y Ciencias Biológicas, Universidad Nacional del Litoral, Santa Fe, Argentina

2 Consejo Nacional de Investigaciones Científicas y Técnicas (CONICET), Ciudad Autónoma de Buenos Aires, Argentina
Contacto de la autora: María Eugenia Oliva

E-mail: meoliva@fbcb.unl.edu.ar

Correspondencia: Ciudad Universitaria, Paraje El Pozo, cc242, FBCB-UNL, (CP3000), Santa Fe, Argentina

Fecha de trabajo recibido: 25/02/19

Fecha de trabajo aceptado: 13/04/19

Conflictos de interés: las autoras declaran que no existe conflicto de interés 


\section{INTRODUCCIÓN}

El síndrome metabólico (SM) se define como un factor de riesgo múltiple para el desarrollo de enfermedad cardiovascular y diabetes mellitus tipo 2 (DM2), patologías asociadas a altas tasas de morbilidad y mortalidad. Este síndrome, de alta prevalencia a nivel mundial, constituye un problema de Salud Pública de principal interés socioeconómico dado que se asocia a un elevado costo de la atención de aquellos que lo padecen y el impacto sobre su calidad de vida. Entre los determinantes de riesgo cardiometabólicos que lo caracterizan se incluyen, entre otros, obesidad, adiposidad visceral, dislipemia, hipertensión arterial y resistencia insulínica (RI). Esta última jugaría un rol patogénico central en el desarrollo de muchos de los desórdenes metabólicos incluidos en el mismo?.

El músculo esquelético (ME) se considera uno de los principales tejidos periféricos involucrados en el mantenimiento de la homeostasis de la glucosa. La insulina cumple un rol clave en este aspecto al estimular la captación de glucosa, la glucólisis y la síntesis de glucógeno en este tejido². El acúmulo de lípidos en las fibras musculares esqueléticas se ha vinculado al desarrollo de lipotoxicidad y $\mathrm{Rl}^{3}$. Al respecto numerosos estudios, tanto en humanos como en animales de experimentación, demostraron que un acúmulo de triglicéridos (TG) intramusculares se encuentra estrechamente asociado a la $\mathrm{RI}^{4}$. Más aún, diferentes trabajos indicaron que intermediarios lipídicos -principalmente derivados de ácidos grasos saturados como los acil-CoA de cadena larga (LCACOA), diacilglicerol (DAG) y ceramidas- serían las especies moleculares involucradas directa o indirectamente en alterar la cascada de señalización de la insulina en este tejido ${ }^{5-8}$.

Sin embargo los mecanismos que contribuirían al desarrollo de la lipotoxicidad en el ME y su asociación con la RI no están completamente esclarecidos. Algunos autores sugieren que una mayor disponibilidad de lípidos (por incremento en los niveles circulantes de ácidos grasos libres y/o TG) y diferentes defectos del metabolismo de los ácidos grasos en este tejido entre los que se incluyen, la captación, transporte, oxidación y esterificación de los mismos, podrían estar involucrados ${ }^{9-10}$. Sumado a esto, factores de transcripción que controlan estos procesos, como el receptor activado por proliferadores peroxisomales $\alpha$ (PPAR $\alpha$ ), jugarían un rol relevante en este aspecto ${ }^{11}$.

Por otra parte, un sensor metabólico clave en el metabolismo de los lípidos y de la glucosa en el ME es la proteína quinasa activada por AMP (AMPK, sus siglas en inglés), que regula además una amplia gama de eventos fisiológicos, incluidos el crecimiento y proliferación celular, la función y biogénesis mitocondrial, y factores relacionados con la resistencia a la insulina como la inflamación y el estrés oxidativo $^{12}$. Asimismo diferentes estudios demostraron que los cambios en los genes y proteínas de la vía inflamatoria podrían contribuir a la disfunción mitocondrial observada en los músculos resistentes a la insulina y conducir a una disminución de la oxidación de las grasas; esto se asociaría al acúmulo de grasa y anomalías en la vía de señalización de la insulina ${ }^{13}$.

Hemos demostrado previamente que la administración crónica de una dieta rica en sacarosa (DRS) (60-65\% de la energía total) a ratas normales induce $\mathrm{RI}$, dislipemia, adiposidad visceral y acúmulo ectópico de lípidos (músculo esquelético/cardíaco, hígado y páncreas) por lo que representa un modelo experimental que mimetiza el fenotipo del SM humano. En el músculo esquelético el incremento del contenido de lípidos se asocia a un progresivo deterioro de la captación/transporte (niveles de GLUT 4), vías oxidativas y no oxidativas de la glucosa. Estos cambios se acompañan de una reducción en los niveles de masa proteica del sustrato receptor de insulina-1 (IRS-1, sus siglas en inglés) $)^{14-16}$.

Teniendo en cuenta que el ME tiene vital importancia en el desarrollo de RI, resulta un importante target para el estudio de la fisiopatología del SM, así como para la futura búsqueda de estrategias terapéuticas para el tratamiento/prevención del mismo.

\section{OBJETIVOS}

El objetivo del presente trabajo fue investigar algunos posibles mecanismos involucrados en la lipotoxicidad del ME y su asociación con la resistencia insulínica en un modelo experimental de dislipemia e insensibilidad insulínica inducido por la administración crónica de DRS.

\section{MATERIALES Y MÉTODOS Animales y dietas}

Se utilizaron ratas macho de la cepa Wistar provenientes del Bioterio de la Facultad de Farmacia y Bioquímica, UBA, Buenos Aires, con un peso inicial aproximado de 180-200 g. Los animales se mantuvieron bajo condiciones ambientales de temperatura $\left(22 \pm 1^{\circ} \mathrm{C}\right)$, aireación, humedad $(55 \pm 5 \%)$ controladas y un ciclo de luz-oscuridad de 12 h (7-19 h), tenien- 
do libre acceso al agua y a una dieta comercial de laboratorio (GEPSA FEED, Buenos Aires, Argentina). Luego de una semana de aclimatación los animales se dividieron aleatoriamente en dos grupos. El grupo control (DC) $(n=12)$ recibió una dieta semi sintética (\% en peso): almidón (62,5), aceite de maíz (8) y proteínas (17). El grupo experimental (DRS) $(n=12)$ recibió la misma dieta semi sintética donde la sacarosa sustituyó al almidón como fuente de hidratos de carbono. Los animales de ambos grupos fueron alimentados por seis meses. La Tabla 1 muestra la composición de las dietas cuyos componentes se basaron en las recomendaciones del Comité ad hoc del American Institute of Nutrition ${ }^{17}$. Las mismas se prepararon semanalmente y se mantuvieron a $4^{\circ} \mathrm{C}$ hasta el momento del consumo. Ambas proveyeron aproximadamente $16,30 \mathrm{KJ} / \mathrm{g}$ de comida y se administraron ad libitum. Durante todo el período experimental se registraron el peso corporal y la ingesta energética de las ratas pertenecientes a cada grupo dietario. El protocolo experimental fue evaluado y aprobado por el Comité de Ética y Seguridad de Investigación de la Facultad de Bioquímica y Ciencias Biológicas (UNL), Santa Fe, Argentina.

\begin{tabular}{|l|c|c|c|c|}
\hline Componentes & \multicolumn{2}{|c|}{ DC } & \multicolumn{2}{c|}{ DRS } \\
\hline & $\%$ en peso & $\%$ en KJ & $\%$ en peso & $\%$ en peso \\
\hline Almidón & 62,5 & 65,8 & - & - \\
\hline Sacarosa & - & - & 62,5 & 65,8 \\
\hline Caseína & 17 & 17,4 & 17 & 17,4 \\
\hline Aceite de maíz & 8 & 16,8 & 8 & 16,8 \\
\hline Vitaminas $^{\mathrm{b}}$ & 1 & & 1 & \\
\hline Fibras & 7,5 & & 7,5 & \\
\hline Sales & & & 3,5 & \\
\hline Colina bitartrato $^{\mathrm{c}}$ & 0,2 & & 0,2 & \\
\hline Metionina & 0,3 & & 0,3 & \\
\hline
\end{tabular}

a) Las composición de las dietas se basan en las recomendaciones de la dieta AIN-93 con modificaciones.

b) Mezcla de vitaminas AIN-93M-VX (g/kg de mezcla): vitamina A (500.000 UI/g) 0,8; vitamina D3 (400.000 UI) 2,75; vitamina $E$ (5.000 UI) 15,0; vitamina K 0,075; D-Biotina 0,02; vitamina B12 0,0025; ácido fólico 0,20; niacina 3,0; pantotenato de calcio 1,6;

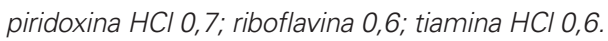

c) Mezcla de sales AIN-93M-MX (g/kg de mezcla): carbonato de calcio 357,0; fosfato monobásico de potasio 250; cloruro de sodio 74; sulfato de potasio 46,6; citrato de potasio monohidrato 28,0; óxido de magnesio 24,0; citrato férrico 6,06; carbonato de zinc 1,65; carbonato de manganeso 0,63; carbonato cúprico 0,30; yodato de potasio 0,01; selenato de sodio 0,01025; molibdato de amonio 0,00795; cromato de potasio 0,275.

Tabla 1: Composición de las dietas experimentales ${ }^{a}$.

\section{Métodos analíticos}

Finalizado el período experimental, el alimento fue removido al final del período de oscuridad y los animales ( $n=6$ de cada grupo) fueron anestesiados con pentobarbital sódico (60 mg/kg peso corporal) administrado por vía intraperitoneal. Se obtuvieron muestras de sangre de la vena cava inferior y se centrifugaron rápidamente. El suero obtenido se utilizó inmediatamente o conservó a $-20^{\circ} \mathrm{C}$. El músculo esquelético gastrocnemio rápidamente se removió y congeló a $-80^{\circ} \mathrm{C}$ hasta su procesamiento.

Los niveles séricos de TG y glucosa se cuantificaron con kits enzimáticos comerciales (Wiener Lab., Rosario, Argentina). Los ácidos grasos no esterificados (AGNE) se determinaron con un kit comercial (Randox, Reino Unido) basado en el método enzimático colorimétrico acil-CoA sintetasa/ acil-CoA oxidasa. La insulinemia se determinó según se describió en trabajos previos ${ }^{15}$. Los niveles de adiponectina y TNF- $\alpha$ en suero se midieron con kits comerciales de ELISA (Thermo Scientific, Rockford, EE.UU.).

\section{Clamp euglucémica hiperinsulinémica}

En otro grupo de animales pertenecientes a ambos lotes se midió la sensibilidad insulínica a través de la técnica clamp euglucémica hiperinsulinémica. Para ello, a animales con $5 \mathrm{~h}$ de ayuno ( $n=6$ de cada grupo) se les realizó una infusión de insulina humana recombinante (Humulin R, Lilly, Indianápolis, EE.UU.) a 0,8 U/kg peso corporal/h durante $120 \mathrm{~min}$. La glucemia se mantuvo a un nivel euglucémico (5,5-6,0 mM) mediante la infusión de glucosa a velocidad variable. La velocidad de infusión de glucosa VIG -mg glucosa infundida calculada durante los últimos 60 minutos de la prueba (estado estacionario)- es una medida de la sensibilidad periférica a la insulina. Se describieron detalles de la metodología en trabajos previos $^{18}$. Los resultados se expresaron como $\mathrm{mg}$ glucosa $/ \mathrm{kg}^{*} \mathrm{~min}$.

\section{Determinaciones en músculo esquelético}

El contenido de TG, LCACoA y DAG se determinó en homogenatos de músculo esquelético gastrocnemio según se describió en trabajos previos del grupo ${ }^{15,16,19}$.

\section{Actividad enzimática M-CPT}

Las actividades de las enzimas M-CPT1, M-CPT2 y M-CPT total se midieron espectrofoto- 
métricamente según la metodología descripta por Ling et $a .^{20}$ con breves modificaciones y utilizada en trabajos previos del grupo ${ }^{21}$. El tejido congelado fue homogenizado a $4^{\circ} \mathrm{C}$ en buffer de homogenización conteniendo: HEPES $20 \mathrm{mM}, \mathrm{KCl} 140 \mathrm{mM}$, EDTA $10 \mathrm{mM}, \mathrm{MgCl}_{2} 5 \mathrm{mM}(\mathrm{pH} 7,4)$ y se centrifugó a $500 \mathrm{xg}$ a $4^{\circ} \mathrm{C}$ durante $10 \mathrm{~min}$. El sobrenadante obtenido se centrifugó a $4^{\circ} \mathrm{C}$ a $9.000 \mathrm{xg}$ por 45 min y el pellet mitocondrial resultante se resuspendió en buffer de homogenización. Para determinar la actividad M-CPT total, una alícuota del extracto mitocondrial obtenido se adicionó a un medio de reacción compuesto por: HEPES $20 \mathrm{mM}$ (pH:7,4), EGTA $1 \mathrm{mM}$, sacarosa $220 \mathrm{mM}, \mathrm{KCl}$ 40 mM, DTNB 0,1 mM, albúmina de suero bovino 1,3 mM y palmitoil-CoA $40 \mu \mathrm{M}$. La reacción se inició con el agregado de L-carnitina (concentración final $1 \mathrm{mM}$ ). La formación de TNB-CoAS se monitoreó continuamente a $412 \mathrm{~nm}$ durante $5 \mathrm{~min}$ a $37^{\circ} \mathrm{C}$. La actividad M-CPT total se calculó en base a la variación de la absorbancia por minuto y al coeficiente de extinción molar del DTNB $(\varepsilon=13,6 \mathrm{mM})$. La actividad de la M-CPT2 se determinó en las mismas condiciones de ensayo en presencia de malonil-CoA10 $\mu \mathrm{M}$ (inhibidor de la M-CPT1) en el medio de reacción. La actividad M-CPT1 se calculó como la diferencia entre la M-CPT total y M-CPT2. Los resultados se expresaron como $\mathrm{nmol} / \mathrm{min} \times \mathrm{mg}$ de proteína. La concentración de proteínas en una alícuota del extracto mitocondrial se determinó por el método de Bradford (Sigma).

\section{Niveles de masa proteica de $\operatorname{PPAR} \alpha$, AMPK y AMPKp}

Los niveles de masa proteica de PPAR $\alpha$, AMPK y AMPK fosforilada (AMPKp) (Thr172) se midieron por la técnica de Western Blot en extractos proteicos de tejido muscular obtenidos como se describió anteriormente en trabajos del grupo ${ }^{21,22}$. Las proteínas, cuantificadas mediante el ensayo de Bradford (SIGMA), se separaron por SDS-PAGE y se transfirieron a membranas de PVDF. Para el inmunobloting las membranas se expusieron con anticuerpos primarios específicos: policlonal anti-
PPAR $\alpha$, anti-AMPK o anti-AMPKp (Thr172) (Santa Cruz Biotechnology, Inc.). Luego se incubaron con el anticuerpo secundario (ISAL-FBCB-UNL) conjugado con horseradish-peroxidase durante $2 \mathrm{~h}$, con agitación y a temperatura ambiente. Las bandas se visualizaron por quimioluminiscencia de acuerdo a las instrucciones del fabricante (Super Signal West Pico Chemiluminiscent Substrate, Pierce Biotechnology, Rockford, IL). La intensidad de las bandas se cuantificó con el software para capturar imágenes Scion Image versión 4.0.

La relación entre la cantidad de muestra procesada en el inmunoblotting y la intensidad de la señal observada fue lineal bajo las condiciones previamente descriptas. Los niveles de la masa proteica de la proteína en estudio se normalizaron con los niveles de la masa proteica del marcador $\beta$ actina (Santa Cruz Biotechnology, Inc., Santa Cruz, CA, EE.UU.).

\section{Análisis estadístico}

Los resultados se expresaron como media \pm SEM. Las comparaciones estadísticas se realizaron transversalmente entre ambos grupos dietarios. La distribución normal de los datos y la homogeneidad de las varianzas se comprobó con el test de Shapiro-Wilk y test de Levene respectivamente. La significancia estadística entre ambos grupos (DC y DRS) se determinó mediante la prueba t de Student. Valores de $p<0,05$ se consideraron estadísticamente significativos (SPSS 17.0 para Windows, SPSS INC. Chicago, Illinois) $)^{23}$. Todos los valores $\mathrm{p}$ reportados fueron de dos colas.

\section{RESULTADOS}

\section{Ganancia de peso e ingesta energética}

La Figura 1 muestra la ganancia de peso e ingesta energética de los animales pertenecientes a los diferentes grupos en estudio. Al corroborar resultados previos del grupo ${ }^{15,18}$, encontramos un significativo incremento en la ganancia de peso e ingesta energética en el lote de animales que consumió DRS comparado con los controles de igual edad $(p<0,05)$. 

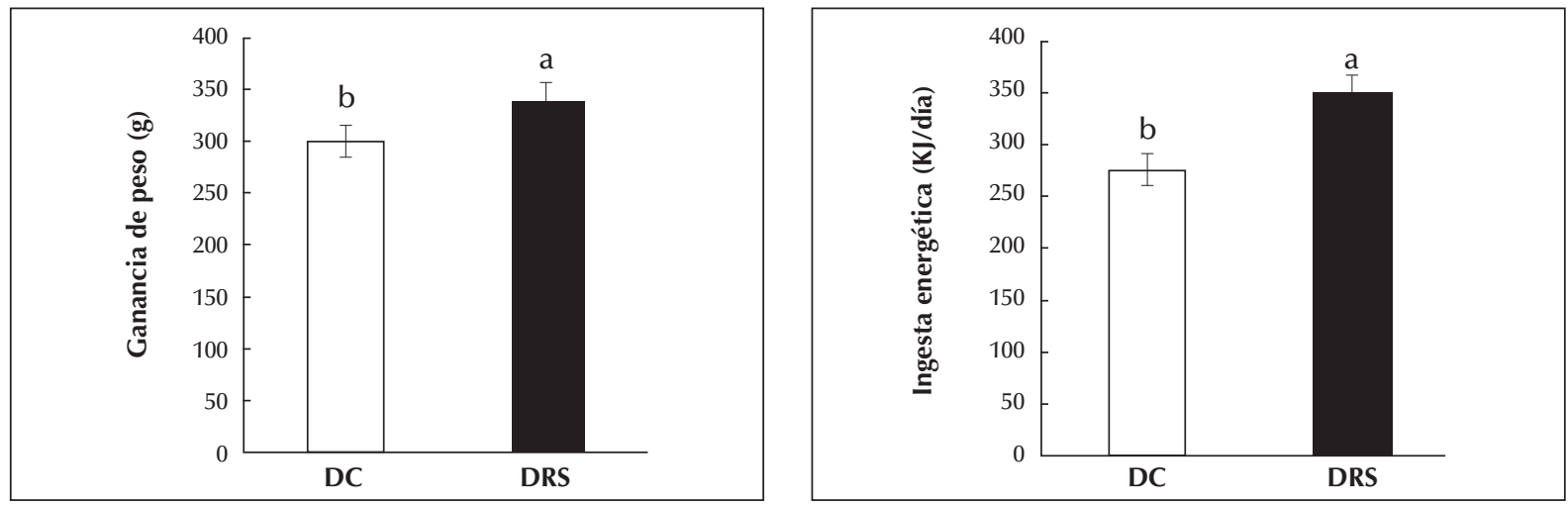

Los valores se expresaron como media \pm SEM. Los valores que no comparten la misma letra son significativamente diferentes $(p<0,05)$.

Figura 1: Ganancia de peso e ingesta energética de ratas alimentadas con dieta control (DC) y dieta rica en sacarosa (DRS).

\section{Metabolitos e insulina en suero, contenido de lípidos en músculo esquelético y sensibilidad insulínica}

Corroborando los resultados previos de nuestro grupo ${ }^{15,16}$ encontramos que los niveles de glucosa en suero fueron significativamente mayores en las ratas alimentadas durante seis meses con DRS cuando se compararon con los animales de igual sexo y edad alimentados con DC (glucosa [mM]: DC: $6,4 \pm 0,2 ;$ DRS: $8,3 \pm 0,3 ; p<0,05)$. Los niveles de insulina fueron similares en ambos grupos dietarios (insulina [pM]: DC: $395,0 \pm 30,5$; DRS: 408,0 $\pm 28,5$ ).

Los niveles de lípidos (TG y AGNE) determinados en suero y los lípidos intramusculares (TG, LCACoA y DAG) de los animales de los lotes DC y DRS al final del período experimental (seis meses) se detallan en la Tabla 2. Como se describió previamente ${ }^{15,18,19}$, los animales alimentados con DRS presentaron un incremento significativo en los niveles de TG y AGNE en suero y de los lípidos intramusculares (TG, LCACoA y DAG) en comparación con los animales alimentados con DC.

Además en el grupo DRS se observó un marcado deterioro de la sensibilidad insulínica periférica global en comparación con los animales con-

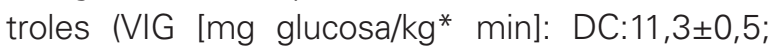
DRS:4,5 $\pm 0,4 ; p<0,05)$.

\begin{tabular}{|l|c|c|}
\hline & DC & DRS \\
\hline Suero & & \\
\hline TG $(\mathrm{mM})$ & $0,68 \pm 0,06^{\mathrm{b}}$ & $1,78 \pm 0,12^{\mathrm{a}}$ \\
\hline AGNE $(\mu \mathrm{M})$ & $316,0 \pm 20,2^{\mathrm{b}}$ & $713,0 \pm 20,8^{\mathrm{a}}$ \\
\hline Músculo esquelético & & \\
\hline TG $(\mu \mathrm{mol} / \mathrm{g}$ TH) & $3,50 \pm 0,20^{\mathrm{b}}$ & $7,20 \pm 0,30^{\mathrm{a}}$ \\
\hline LCACoA $(\mu \mathrm{mol} / \mathrm{g}$ TH) & $5,9 \pm 0,5^{\mathrm{b}}$ & $11,9 \pm 0,8^{\mathrm{a}}$ \\
\hline DAG $(\eta \mathrm{mol} / \mathrm{g}$ TH) & $109,2 \pm 12,5^{\mathrm{b}}$ & $204,3 \pm 19,4^{\mathrm{a}}$ \\
\hline
\end{tabular}

Los valores se expresaron como media \pm SEM. Los valores en una línea que no presentan la misma letra superíndice son significativamente diferentes $(p<0,05)$.

Tabla 2: Niveles de lípidos en suero y músculo esquelético en ratas alimentadas con dieta control (DC) y dieta rica en sacarosa (DRS).

\section{Actividad enzimática M-CPT total, M-CPT1 y M-CPT2, y masa proteica del PPAR $\alpha$}

En la Tabla 3 se observa que la actividad M-CPT total se encontró significativamente disminuida $(p<0,05)$ en el lote de animales que consumió DRS al compararlo con aquellos que recibieron DC. Un patrón similar se observó al evaluar la actividad M-CPT1. No se hallaron cambios en la actividad 
enzimática M-CPT2 entre los diferentes grupos dietarios. El análisis cuantitativo de los inmunoblots mostró que la abundancia relativa del PPAR $\alpha$ estaba significativamente disminuida $(p<0,05)$ en los animales alimentados crónicamente (seis meses) con DRS comparado con los animales alimentados con DC (Figura 2).

\begin{tabular}{|l|c|c|}
\hline & DC & DRS \\
\hline M-CPT total (nmol/min x mg proteína) & $12,20 \pm 0,30^{\text {a }}$ & $10,40 \pm 0,50^{\text {b }}$ \\
\hline M-CPT1 (nmol/min x mg proteína) & $3,75 \pm 0,20$ & $2,86 \pm 0,30$ \\
\hline M-CPT2 (nmol/min x mg proteína) & $7,95 \pm 0,50$ & $7,05 \pm 0,35$ \\
\hline
\end{tabular}

Los valores se expresaron como media \pm SEM. Los valores que no comparten la misma letra son significativamente diferentes $(p<0,05)$.

Tabla 3: Actividad enzimática M-CPT total, M-CPT1 y M-CPT2 en músculo esquelético gastrocnemio de ratas alimentadas con DC y DRS.

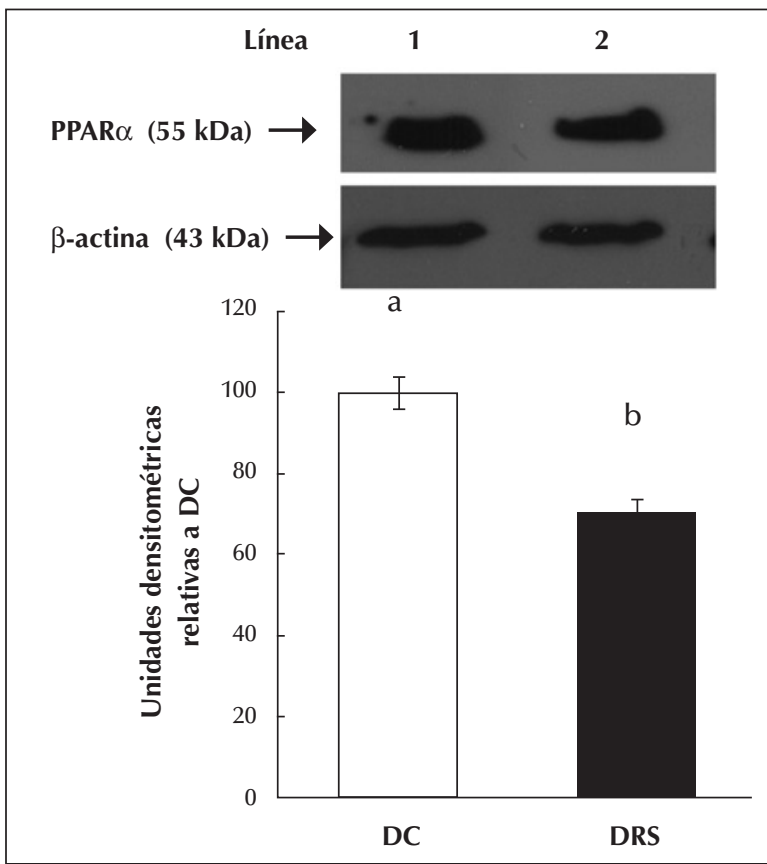

Los valores se expresan como media \pm SEM relativos a $D C$ y corregidos por los niveles de $\beta$ actina presentes en cada una de las muestras (se utilizaron seis animales en cada grupo experimental). Las diferentes letras muestran valores significativamente diferentes $(p<0,05)$.

Figura 2: Panel superior: inmunoblots del PPAR $\alpha$ en músculo esquelético gastrocnemio de ratas alimentadas con DC y DRS. Línea 1: DC; Línea 2: DRS. Panel inferior: análisis densitométrico de la masa de proteica del PPAR $\alpha$ en músculo gastrocnemio de ratas alimentadas con DC y DRS.

la sensibilidad insulínica (VIG) comparado con los animales controles.

Se ha demostrado que los niveles elevados de TNF $\alpha$ y los bajos niveles de adiponectina en plasma tienen un papel directo en la $\mathrm{RI}$ del músculo esquelético ${ }^{24,25}$. Las Figuras $3 \mathrm{~B}$ y $3 \mathrm{C}$ muestran la existencia de una correlación negativa entre los niveles de AMPKp en el músculo esquelético y los niveles séricos de TNF $\alpha$, y una correlación positiva con los niveles de adiponectina sérica. En los animales alimentados con DRS los menores niveles de masa proteica de AMPKp se asociaron a altos niveles de TNF $\alpha$ y bajos niveles de adiponectina en suero en comparación con los animales controles. 


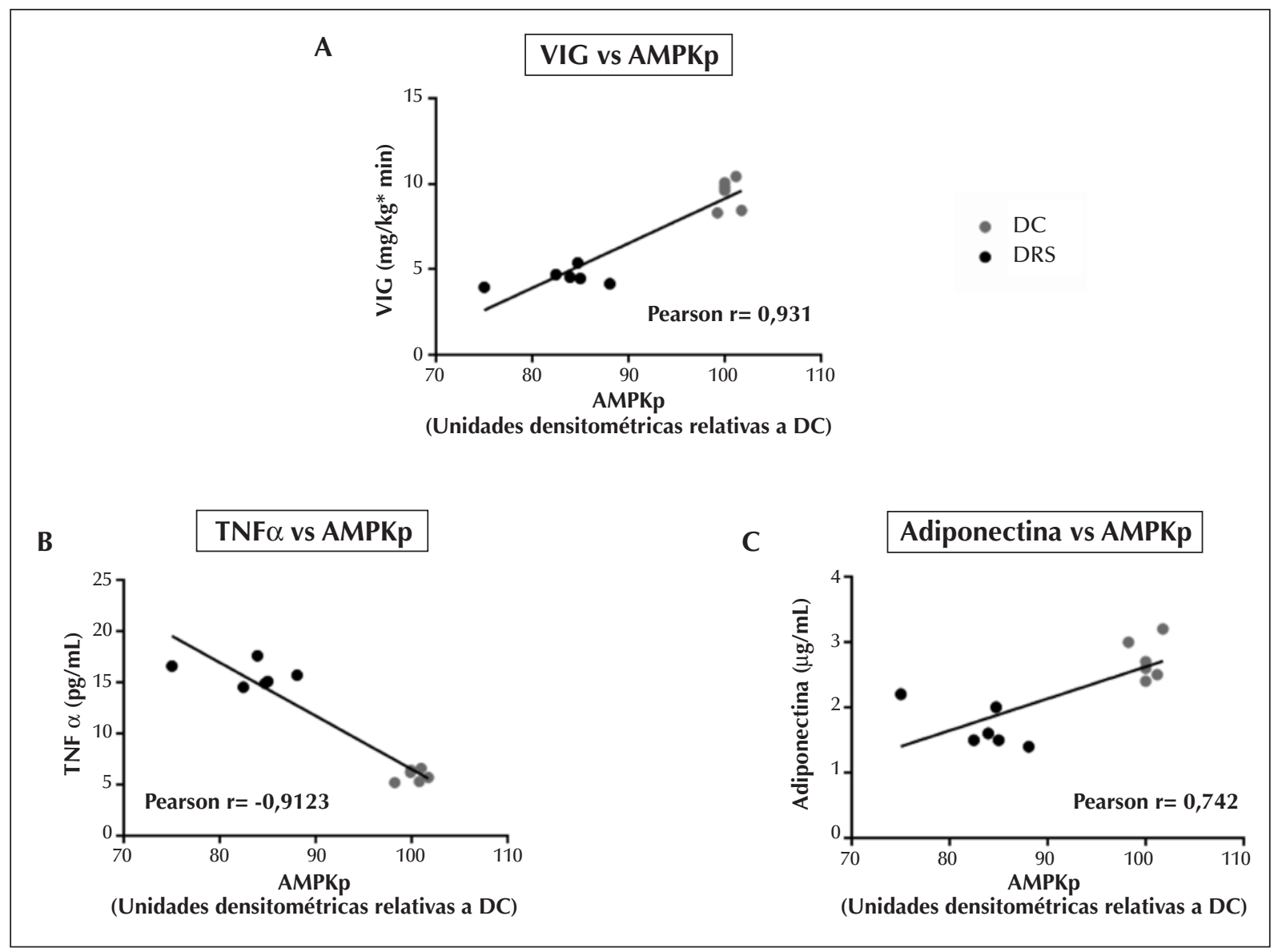

Figura 3 (A, B y C): Correlaciones de los niveles de masa proteica de AMPKp en músculo esquelético con la sensibilidad insulínica (VIG) (A), y niveles séricos de TNF $\alpha$ (B) y adiponectina (C) en ratas alimentadas con dieta control (DC) y dieta rica en sacarosa (DRS).

\section{DISCUSIÓN}

El presente trabajo analizó algunas vías metabólicas implicadas en el acúmulo de lípidos en músculo esquelético (lipotoxicidad) y su asociación con la resistencia insulínica en ratas macho Wistar alimentadas crónicamente con una DRS. La dislipemia, moderada hiperglucemia y resistencia insulínica periférica global presente en el modelo experimental se asociaron con los siguientes hallazgos: 1) el acúmulo de especies lipídicas en músculo esquelético se acompañó de una disminución de la actividad enzimática M-CPT total y M-CPT1 y de la masa proteica del PPAR $\alpha$; 2) una disminución de la masa proteica de la AMPKp se asoció con bajos niveles de adiponectina y elevados niveles de TNF $\alpha$ en suero.

El depósito de lípidos en el ME se encuentra estrechamente asociado con el desarrollo de RI. Al respecto se ha propuesto que un desbalance entre el flujo de lípidos hacia el tejido muscular y la capacidad oxidativa del mismo cumplirían un rol clave en la lipotoxicidad y RI ${ }^{3}$. Trabajos previos del grupo demostraron en animales alimentados crónicamente con DRS una mayor disponibilidad de lípidos plasmáticos (TG, AGNE), consecuencia de un incremento en la síntesis y secreción hepática de VLDL-TG y menor remoción plasmática de los mismos. Además el tejido adiposo de estos animales presenta hipertrofia de los adipocitos con aumento de la lipolisis y menor acción antilipolítica de la insulina lo que conduce a una mayor liberación de ácidos grasos libres a la circulación. Todo esto favorece al acúmulo ectópico de lípidos en tejidos no adiposos 26,27 .

En este sentido en el presente trabajo hallamos, en los animales dislipémicos insulinorresistentes alimentados con DRS, que el acúmulo de especies lipídicas (TG, LCACoA y DAG) en ME se acompañó de una menor actividad enzimática M-CPT total asociada a un descenso de la actividad 
M-CPT1, enzima clave en el control de la oxidación mitocondrial de ácidos grasos, siendo éste un posible mecanismo involucrado en el desarrollo de la lipotoxicidad. En línea con estos resultados, Bi et al. ${ }^{28}$ observaron en ratas con DM2 inducida por administración de una dieta rica en grasa y bajas dosis de estreptozotocina, que el acúmulo de TG en músculo esquelético se acompañó de una reducción $(47 \%)$ de los niveles de masa proteica de la enzima CPT-1, demostrando la existencia de una correlación negativa entre estos parámetros. A su vez, Perdomo et al. ${ }^{29}$ describieron en células musculares L-6 cultivadas en presencia de palmitato, un acúmulo de especies lipídicas (TG, DAG y ceramidas) y menor sensibilidad a la insulina. La inducción de la sobreexpresión de la enzima CPT-1 en estas células logró mejorar la sensibilidad insulínica de las mismas. Asimismo Kim et al. ${ }^{30}$ observaron una disminución en la actividad de la CPT-1 y otras enzimas mitocondriales tales como citrato sintasa y $\beta$ hydroxyacyl-CoA deshidrogenasa en el músculo esquelético de mujeres obesas.

Por otra parte, nuestros resultados mostraron una disminución en los niveles del factor de transcripción PPAR $\alpha$ en músculo esquelético de animales alimentados con DRS. En este tejido se ha visto que el PPAR $\alpha$ juega un rol importante en el control transcripcional de la oxidación de ácidos grasos al estimular la expresión de genes involucrados en la misma, entre ellos la CPT-1 ${ }^{31}$. En este sentido, Li et al. ${ }^{32}$ observaron una disminución significativa en los niveles de ARNm del PPAR $\alpha$ en el músculo esquelético de hamsters obesos insulinorresistentes alimentados con dieta alta en grasa, lo cual se asoció con un descenso en los niveles de ARNm de la CPT-1 y acúmulo de TG en el tejido. Además Kim et al. ${ }^{33}$ observaron en ratas macho Sprague-Dawley alimentadas con dieta rica en grasas durante ocho semanas una reducción en los niveles de ARMm del PPAR $\alpha$ y CPT-1 en músculo esquelético.

Diferentes autores demostraron una disminución en la actividad de AMPK en modelos genéticos de roedores con fenotipo de SM, incluidos los ratones ob/ob, ratas fa/fa y rata macho ZDF. Se sabe que la AMPK cumple un rol clave en el control de los niveles de malonil-CoA, principal inhibidor de la CPT-1. Niveles incrementados de malonil-CoA inhiben la captación y oxidación de ácidos grasos ${ }^{13}$. Al respecto, Bandyopadhyay et al. ${ }^{34}$ encontraron una disminución en la actividad de la AMPK en el músculo de sujetos insulinorresistentes, lo cual se acompañó de una reducción en la oxidación de ácidos grasos y acúmulo de TG y acil-CoA de cadena larga. En el presente trabajo encontramos niveles disminuidos en la masa proteica de la AMPKp en los animales alimentados con DRS asociados con una disminución de los parámetros relacionados con la oxidación de ácidos grasos (CPT-1 y PPAR $\alpha$ ).

Diferentes estudios sugieren un rol protagónico de las citoquinas (entre ellas TNF $\alpha$ y adiponectina) en la regulación de la AMPK. Al respecto, Steinberg et al. ${ }^{35}$ han demostrado en estudios in vivo e in vitro que la resistencia insulínica inducida por TNF $\alpha$ disminuye la actividad de la AMPK por desfoforilación de la misma, vía regulación transcripcional de la proteína fosfatasa 2. Esto se acompaña de una supresión de la oxidación de ácidos grasos y acúmulo de TG y DAG intramuscular.

Diferentes autores sugieren que la adiponectina sería un regulador de la bioenergética mitocondrial en el músculo esquelético a través de la activación de la AMPK, con lo cual una disminuida señal de la adiponectina podría deteriorar la capacidad oxidativa de los lípidos e incrementar el acúmulo intramuscular de los mismos ${ }^{36}$. Yoon et al. ${ }^{37}$, en miocitos C2C12, observaron que la adiponectina incrementa la oxidación de ácidos grasos a través de la activación secuencial de la AMPK, la p38MAK y PPAR $\alpha$. Más aún, Chen et al. ${ }^{38}$ demostraron en miotubos humanos que la adiponectina induce la oxidación de los lípidos a través de la activación de la AMPK y que este mecanismo se encuentra deteriorado en miotubos de individuos obesos con DM2.

En nuestro trabajo encontramos, en los animales alimentados con DRS, que los niveles disminuidos de AMPKp en el músculo esquelético se correlacionan con un aumento de TNF $\alpha$ y un descenso de adiponectina en suero, siendo otro posible mecanismo involucrado en el desarrollo de la lipotoxicidad e insulinorresistencia en el modelo experimental empleado.

\section{CONCLUSIONES}

La prevalencia de SM ha aumentado considerablemente y actualmente es una "epidemia" a nivel mundial que constituye un problema de Salud Pública dado que se asocia a un elevado costo de la atención de aquellos que lo padecen y el impacto sobre su calidad de vida. Si bien la extrapolación de los resultados obtenidos en modelos animales hacia el 
humano debe ser muy cuidadosa, este trabajo pretende contribuir al conocimiento de los mecanismos que podrían estar involucrados en el desarrollo de la lipotoxicidad y la resistencia insulínica en músculo esquelético inducidos nutricionalmente.

El presente estudio fue financiado por subsidios del Consejo Nacional de Investigaciones Científicas y Técnicas (CONICET) (Grant PIP \# 1122015 0100023CO) y Universidad Nacional del Litoral (CAl+D \# 50420150100011 LI).

\section{BIBLIOGRAFÍA}

1. Bruce $\mathrm{K}$, Hanson M. The developmental origins, mechanisms, and implications of metabolic syndrome. J Nutr 2010; 5:648-52.

2. Carnagarin R, Dharmarajan AM, Dass CR. Molecular aspects of glucose homeostasis in skeletal muscle. A focus on the molecular mechanisms of insulin resistance. Mol Cell Endocrinol 2015; 417:52-62.

3. Turcotte LP, Fisher JS. Skeletal muscle insulin resistance: roles of fatty acid metabolism and exercise. Phys Ther 2008; 88(11): 1-18

4. McGarry JD. Dysregulation of fatty acid metabolism in the etiology of type 2 diabetes. Diabetes 2002; 51: 7-18.

5. Amati F, Dubé JJ, Alvarez-Carnero E, et al. Skeletal muscle triglycerides, diacylglycerols, and ceramides in insulin resistance: another paradox in endurance-trained athletes? Diabetes 2011; 60: 2588-97.

6. Chavez JA, Knotts TA, Wang LP, et al. A role for ceramide, but not diacylglycerol, in the antagonism of insulin signal transduction by saturated fatty acids. J Biol Chem 2003; 278(12): 10297-303.

7. Itani SI, Ruderman NB, Schmieder F, et al. Lipid-induced insulin resistance in human muscle is associated with changes in diacylglycerol, protein kinase $C$, and $1 \kappa B-\alpha$. Diabetes 2002; 51 : 2005-11.

8. Turner N, Kowalski GM, Leslie SJ, et al. Distinct patterns of tissue-specific lipid accumulation during the induction of insulin resistance in mice by high-fat feeding. Diabetologia 2013; 56(7): 1638-48.

9. Holloway GP, Luiken JJ, Glatz JFC, et al. Contribution of FAT/ CD36 to the regulation of skeletal muscle fatty acid oxidation: an overview. Acta Physiol 2008; 194(4): 293-309.

10. Kiens B. Skeletal muscle lipid metabolism in exercise and insulin resistance. Physiol Rev 2006;86(1): 205-43.

11. Burri $L$, Thoresen $H$, Berge RK. The role of PPAR $\alpha$ activation in liver and muscle. PPAR Res 2010; pii: 542359. Doi: $10.1155 / 2010 / 542359$.

12. Lyons $\mathrm{C}$, Roche $\mathrm{H}$. Nutritional modulation of AMPK. Impact upon metabolic-inflammation. Int J Mol Sci 2018; 19: 3092-109.

13. Ruderman N, Carling D, Prentki M, et al. AMPK, insulin resistance, and the metabolic syndrome. J Clin Invest 2013; 123 (7): 2764-72.

14. Chicco A, Soria A, Gutman R, et al. Multiphasic metabolic changes in the heart of rats fed a sucrose-rich diet. Horm Metab Res 1994; 26: 397-403.
15. Chicco A, D'Alessandro ME, Karabatas R, et al. Muscle lipid metabolism and insulin secretion are altered in insulin-resistant rats fed a high sucrose diet. J Nutr 2003; 133(1): 127-33.

16. D’Alessandro ME, Chicco A, LombardoYB. Fish oil reverses the altered glucose transporter, phosphorylation, insulin receptor substrate-1 protein level and lipid contents in the skeletal muscle of sucrose-rich diet fed rats. PLEFA 2012; 88(2): 171-77.

17. Reeves Nielsen FH, Fahey GC. AIN-93 Purified diets for laboratory rodents: final report of the American Institute of Nutrition ad hoc writing Committee on the Reformulation of the AIN-76 ${ }^{\text {a }}$ Rodent Diet. J Nutr 1993; 123(11): 1939-51.

18. D'Alessandro ME, Chicco A, Karabatas L, et al. Role of skeletal muscle on impaired insulin sensitivity in rats fed a sucroserich diet: effect of moderate levels of dietary fish oil. J Nutr Biochem 2000; 11(5):273-80.

19. D'Alessandro ME, Chicco AG, Lombardo YB. A long-term sucrose-rich diet increases diacylglycerol content and membrane $\mathrm{nPKC} \theta$ expression and alters glucose metabolism in skeletal muscle of rats. Nutrition Research 2006; 26(6): 289-296.

20. Ling B, Aziz C, Alcorn J. Systematic evaluation of key L-carnitine homeostasis mechanisms during postnatal development in rat. Nutr Metab (Lond.) 2012; 9:66.

21. Creus A, Ferreira M, Oliva M, et al. Mechanisms involved in the improvement of lipotoxicity and impaired lipid metabolism by dietary $\alpha$-linolenic acid rich Salvia hispanica $L$ (Salba) seed in the heart of dyslipemic insulin-resistant rats. J Clin Med 2016; 28: 5(2). Doi: 10.3390/jcm5020018.

22. Oliva ME, Creus A, Ferreira MR, et al. Dietary soya protein improves intra-myocardial lipid deposition and altered glucose metabolism in a hypertensive, dyslipidaemic, insulin-resistant rat model. Br J Nutr 2018; 119(2): 131-42.

23. Snedecor GWP, Cochran WG. Statistical methods. Ames (lowa): lowa University Press 1967; 339-350.

24. Wieser V, Moschen A, Tilg H. Inflammation, cytokines and insulin resistance: a clinical perspective. Arch Immunol Ther Exp 2013; 61: 119-25.

25. Ziemke F, Mantzoros C. Adiponectin in insulin resistance: lessons from translational research. Am J Clin Nutr 2010, 91(suppl):258S-61S.

26. Lombardo YB, Chicco A. Effects of dietary polyunsaturated n-3 fatty acids on dyslipidemia and insulin resistance in rodents and humans. A review. J Nutr Biochem 2006; 17:1-13.

27. D'Alessandro ME, Selenscig D, Illesca $P$, et al. Time course of adipose tissue dysfunction associated with antioxidant defense, inflammatory cytokines and oxidative stress in dyslipemic insulin resistant rats. Food Funct 2015; 6(4):1299-1309.

28. Bi $\mathrm{Y}$, Cai $\mathrm{M}$, Liang $\mathrm{H}$, et al. Increased carnitine palmitoyl transferase 1 expression and decreased sterol regulatory elementbinding protein 1c expression are associated with reduced intramuscular triglyceride accumulation after insulin therapy in high-fat-diet and streptozotocin-induced diabetic rats. Metabolism 2009; 58(6):779-86.

29. Perdomo G, Commerford SR, Richard AMT, et al. Increased beta-oxidation in muscle cells enhances insulin-stimulated glucose metabolism and protects against fatty acid-induced insulin resistance despite intramyocellular lipid accumulation. J Biol Chem 2004; 279(26):27177-86.

30. Kim J, Hickner R, Cortright R, et al. Lipid oxidation is reduced in obese human skeletal muscle. Am J Physiol Endocrinol Metab 2000; 279: E1039-E44. 
31. Mandard S, Müller M, Kersten S. Peroxisome proliferator-activated receptor $\alpha$ target genes. Cell Mol Life Sci 2004; 61: 393416.

32. Li G, Liu X, Zhu H, et al. Skeletal muscle insulin resistance in hamsters with diabetes developed from obesity is involved in abnormal skeletal muscle LXR, PPAR and SREBP expression. ExpTher Med 2016;11(6): 2259-69.

33. Kim S, Bang C, GuoY, et al. Anti-obesity effects of aster spathulifolius extract in high-fat diet-induced obese rats. J Med Food 2016; 19(4):353-64

34. Bandyopadhyay G, Yu J, Ofrecio J, et al. Increased malonylCoA levels in muscle from obese and type 2 diabetic subjects lead to decreased fatty acid oxidation and increased lipogénesis; thiazolidinedione treatment reverses these defects. Diabetes 2006; 55: 2277-85.
35. Steinberg GR, Michell BJ, Denderen WJ, et al. Tumor necrosis factor $\alpha$-induced skeletal muscle insulin resistance involves suppression of AMP-kinase signaling. Cell Metabolism 2006; 4: 465-74.

36. Civitarese, A, Ukropcova, B, Carling S et al. Role of adiponectin in human skeletal muscle bioenergetics. Cell Metabolism 2006; 4:75-87.

37. Yoon M, Lee G, Chun J. Adiponectin increases fatty acid oxidation in skeletal muscle cells by sequential activation of AMPactivated protein kinase, p38 mitogen-activated protein kinase, and peroxisome proliferator-activated receptor $\alpha$. Diabetes 2006; 55: 2562-70.

38. Chen M, McAinch A, Macaulay S. Impaired activation of AMPkinase and fatty acid oxidation by globular adiponectin in cultured human skeletal muscle of obese type 2 diabetics. J Clin Endocrinol Metab 2005; 90: 3665-72. 